\title{
Energy Efficiency to Improve Network Life Time Using Particle Swarm Optimization Algorithm
}

S. Kannadhasan ${ }^{1, *}$, S. Deepa ${ }^{2}$ and M. Shanmuganantham ${ }^{3}$

${ }^{1}$ Cheran College of Engineering, Department of Electronics and Communication Engineering, Karur, Tamilnadu, India

${ }^{2}$ Madurai Kamaraj University Evening College, Department of Computer Science, Madurai, Tamilnadu, India

${ }^{3}$ Tamilnadu Government Polytechnic College, Department of Electrical and Electronics Engineering, Madurai, Taminadu, India

\section{Abstract}

Remote Sensor Networks (WSNs) have a developing innovation for different applications in reconnaissance, condition, natural surroundings observing, medicinal services and fiasco administration. It has monitor through environment by using sensing device that means of physical properties. WSN is a network that can transmit and receive through the wireless medium by using the sensor devices for various nodes. There are various base stations to control final destination of data from one place to other place. It includes the dense ad-hoc deployment, dynamic topology, spatial distribution, and Network topology, Graph Theory with constraint the bandwidth, energy life time and memory. Based on the problem size increases, it can require as various efforts by using the optimization techniques. This paper is to distinguish the deficiencies in Wireless Sensor Networks the hubs have transmitted starting with one place then onto the next by utilizing the particle swarm enhancement. PSO calculation is contrasted and the different calculations PCA, Neural system and OPAST. Based on the algorithm, the performance analysis is done on specificity, fault detection and fault coverage. The simulation result shows the energy life time, throughput, packet delivery ratio produces good performance when compared to the other algorithms.

Keywords: WSN, PSO, Throughput, Analysis of Node and Energy Efficiency

Received on 18 February 2020, accepted on 06 April 2020, published on 09 April 2020

Copyright $(C 2020$ S. Kannadhasan et al., licensed to EAI. This is an open access article distributed under the terms of the Creative Commons Attribution licence (http://creativecommons.org/licenses/by/3.0/), which permits unlimited use, distribution and reproduction in any medium so long as the original work is properly cited.

doi: 10.4108/eai.13-7-2018.163974

*Corresponding author. Email:kannadhasan.ece@gmail.com 


\section{Introduction}

WSNs have comprise consists of sensors which are classified for collecting, information, processing, storing and transferring information from one node to another in a wireless manner [1]-[5]. These WSN sensors the nodes work with physical phenomenon have some intelligence through network; each other to sense some data can be processed the sensors through the network. Wireless sensor networks consist of protocols and algorithms with increases the life time of sensor networks. Wireless sensor networks mainly used for temperature, pressure, humidity, noise levels, lighting conditions which is used for broadcast communication that means of point-to-point communication[6]-[10]. Wireless sensor networks have limited by power, energy and network capability.

The WSN applications are classified in three categories:

- Devices Monitoring.

- Network area Monitoring.

- Both Network area and Devices Monitoring.

WSN can be used for low power wireless communication for sensors. WSN are often deployed in inaccessible and inhospitable environments throughout the world. Principal component analysis (PCA) is the most popular methods for data transmitted for wide range of applications [11]-[13]. The various numerical techniques are used to calculate the numerical techniques or cross correlation relationship between the variables in the data variables by using the algorithm [14][15]. Zone-based routing (like OPAST) is an algorithm, that divided the small number of networks zone which is the area covered by the sensor networks.

\section{Structure of the Algorithm}

In this paper, based on the algorithm they address the problem of outlier detection in WSNs. They provide a technique-based algorithm framework to detect the techniques designed for WSNs. The proposed algorithm introduces the node characteristic and description of different detection techniques .Based on the detection techniques to compare the various sensor nodes for detection. This paper proposes a monitoring and diagnosis with dynamic power for applying the WSNs devices for various signal acquisition, processing, and transmission. The Proposed algorithm, the nodes which are responsible received the data collection and transmitting through supervisory controller. DPM protocol is used for sensor nodes to increases the Wireless Sensor Network lifetime; this proposed work presents past and present conditions of the PSO algorithm.

If the nodes are failed for fault detection algorithm, by using particle Swarm Optimization (PSO) is efficient in fault detection when the nodes are enormous. Each node is to be consider the particle and travelling with velocity with function of optimized $\mathrm{P}_{\text {best }}$ (personal best) value with $\mathrm{V}(\mathrm{t})$. $\mathrm{P}_{\text {best }}$ and Gbest value is used to optimize techniques for the network to detect the faulty node. PSO algorithm is used to easy the detect the fault nodes that compare other techniques.

Advantages of PSO over other conventional algorithms are:

1) Easy way to implement the hardware or software.

2) Based on the parameters to check availability of the network.

3) Real, integer and binary domains are also check the parameters using this algorithm.

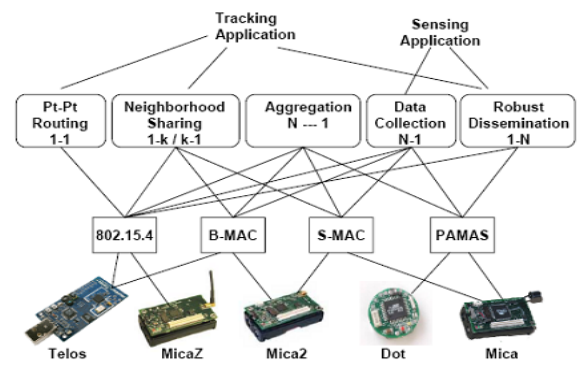

Figure 1. Architecture of WSN

\section{Module of the Proposed Method}

In PSO method, is used to calculate each potential node solution and every solution has positions $\left(\mathrm{T}_{\mathrm{i}, \mathrm{j}}\right)$ and $\left(\mathrm{S}_{\mathrm{i}, \mathrm{j}}\right)$. The solution set of the each solution that means of swarm. Based on the algorithm, each solution is used to generate random numbers from the solution space in the WSN network. Based on the algorithm, the solution of each solution and the swarm is kept as Far best $\left(\mathrm{F}_{\text {besti,j }}\right)$ and Whole best $\left(\mathrm{H}_{\text {besti,j }}\right)$, respectively. Based on the method, the calculation of solution and position of each node is shown in below (1) and (2).

$\mathrm{X}_{\mathrm{i}, \mathrm{j}}(\mathrm{t}+1)=\mathrm{ME}_{\mathrm{i}, \mathrm{j}}(\mathrm{t})+\mathrm{D}_{1} \mathrm{Q}_{1}\left(\mathrm{~F}_{\text {besti,j }}-\mathrm{T}_{\mathrm{i}, \mathrm{j}}(\mathrm{t})\right)+\mathrm{D}_{2} \mathrm{Q}_{2}\left(\mathrm{H}_{\text {besti,j }}-\mathrm{T}_{\mathrm{i}, \mathrm{j}}(\mathrm{t})\right)$

$$
\mathrm{T}_{\mathrm{i}, \mathrm{j}}(\mathrm{t}+1)=\mathrm{T}_{\mathrm{i}, \mathrm{j}}(\mathrm{t})+\mathrm{X}_{\mathrm{i}, \mathrm{j}}(\mathrm{t}+1)
$$

Where $\mathrm{i}$ and $\mathrm{j}$ is the index of the solution and position network. $T$ shows the iteration number, $X_{i, j}(t)$ is the solution of the $\mathrm{i}^{\text {th }}$ and $\mathrm{j}^{\text {th }}$ indexof the particle in the swarm and $\mathrm{T}_{\mathrm{i}, \mathrm{j}}(\mathrm{t})$ is the position. $\mathrm{Q}_{1}$ and $\mathrm{Q}_{2}$ are used to generate the random numbers uniformly distributed between the range of 0 and $1 . \mathrm{D}_{1}$ and $\mathrm{D}_{2}$ are the acceleration numbers. $M$ is the inertial weight. The implementing PSO algorithm for each solution is given below:

1. Each solution has generated random number with suitable dimensional feature.

2. Each particle has calculated by using the formula. 
3. The success of the current solution is better than the success of $F_{\text {besti,j }}$ then determine $F_{\text {besti,j }}$ as the current solution.

4. Current particle is used to determine the success of $\mathrm{G}_{\text {besti,j }}$ is better than the current solution.

5. Calculate the solution and position of the particle using (1) and (2).

6. Follow the steps from 2 to 5 maximum iteration is reached.

Inertial weight is, $M=t_{\max }-t / t_{\max }$

Where $\mathrm{M}$ is inertial weight, $\mathrm{t}$ and $\mathrm{t}_{\max }$ corresponds to the current iteration number and maximum iteration number. The parameters $D_{1}$ and $D_{2}$ are used to determine the particle in terms of local or global solutions in solution space. PSO method is used to identify the iteration that means of constant $D_{1}$ and $D_{2}$ are both equal to 2 for all applications.

\section{Particle Swarm Optimization Algorithm}

PSO Explanation

$\mathrm{Y}$ is the representation of $\mathrm{T}$ in the new basis:

$\mathrm{Y}=\mathrm{PT}$

$\mathrm{R}$ is the covariance matrix in the new basis:

$$
R y=\frac{1}{n-1} Y Y_{T}
$$

Covariance matrix is defined as

- Matrix Defined:

$\mathrm{S}=\mathrm{TT}^{\prime}$

- $\mathrm{S}$ is symmetric.

- Symmetric matrix is used to calculate by an orthogonal matrix of its eigenvectors.

$$
R y=\frac{1}{n-1} Y Y_{T}=\frac{1}{n-1}(P T)(P X T)=\frac{1}{n-1} P X T P X T
$$

Subspace methods have calculated in solving various statistical problems in array signal processing channel estimation and code-division multiple access (CDMA) communications. Based on EVD, subspace methods on same matrices using space decomposition.

\section{Simulation Results and Discussion}

PSO algorithm is used to detect the energy efficiency of best node for the process data transmitted through the network. The proposed techniques, numbers of valid packets are dropped when the rate is increased in the malicious nodes shows the increase in throughput of the network. The numbers of packets are received when the data is transmitted to the sink successfully. The Proposed techniques are increased in throughput of the network.

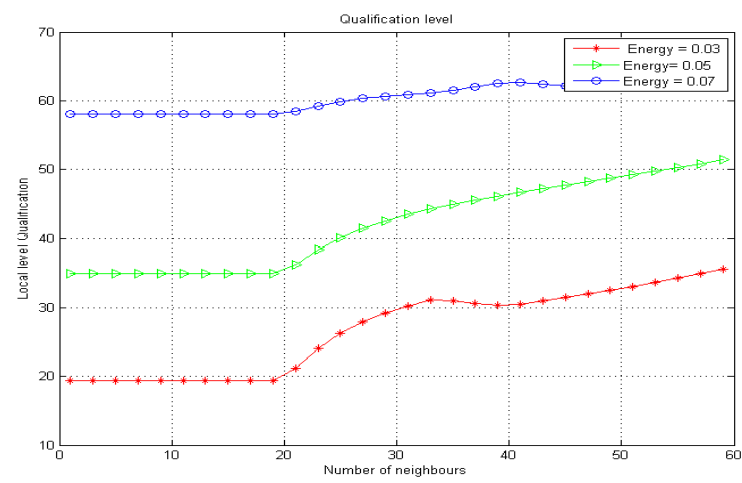

Figure 2. Analysis of Neighbour Node

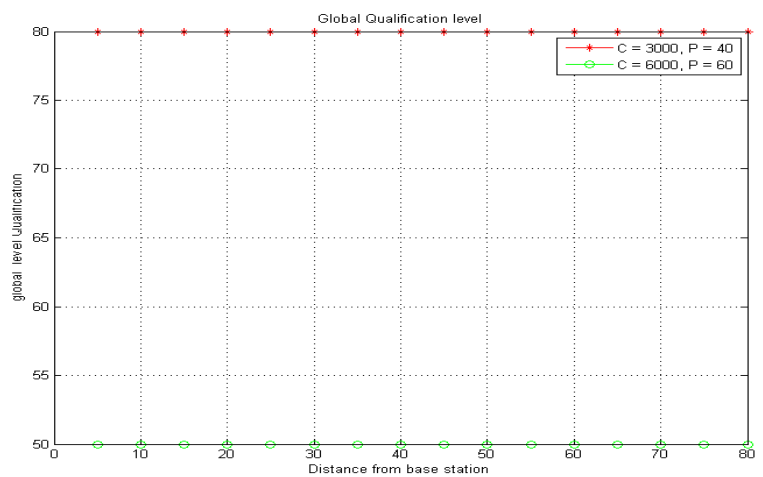

Figure 3. Analysis of Distance of Various Nodes

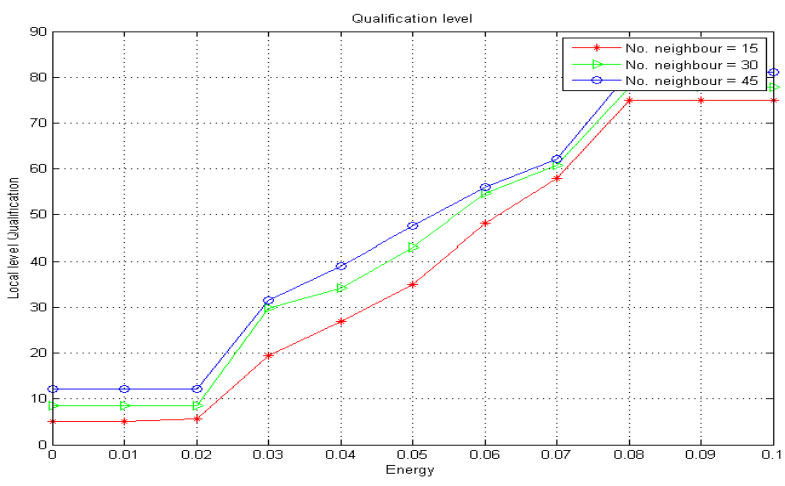

Figure 4. Analysis of Distance of Various Nodes based PSO 


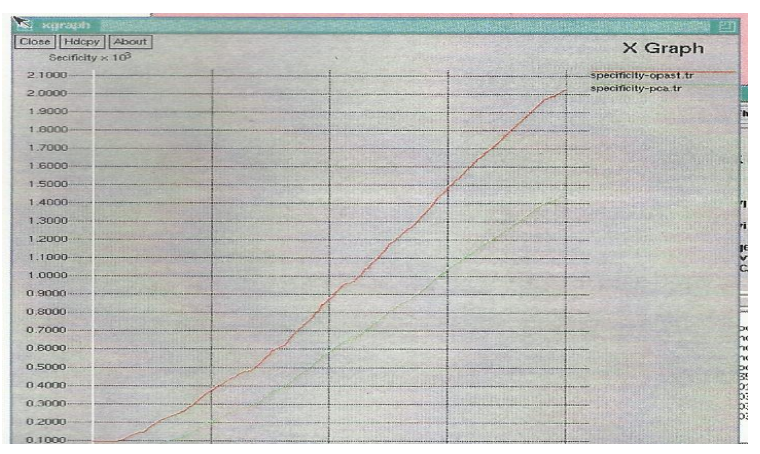

Figure 5. Packet Delivery Ratio

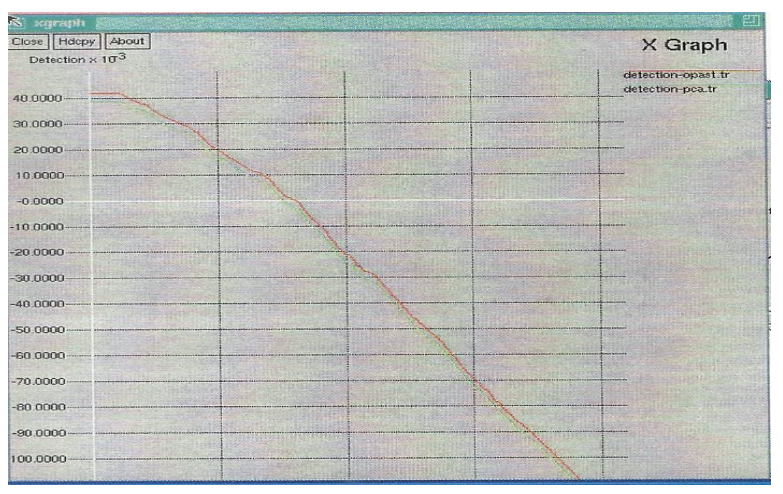

Figure 6. Average Energy Consumption

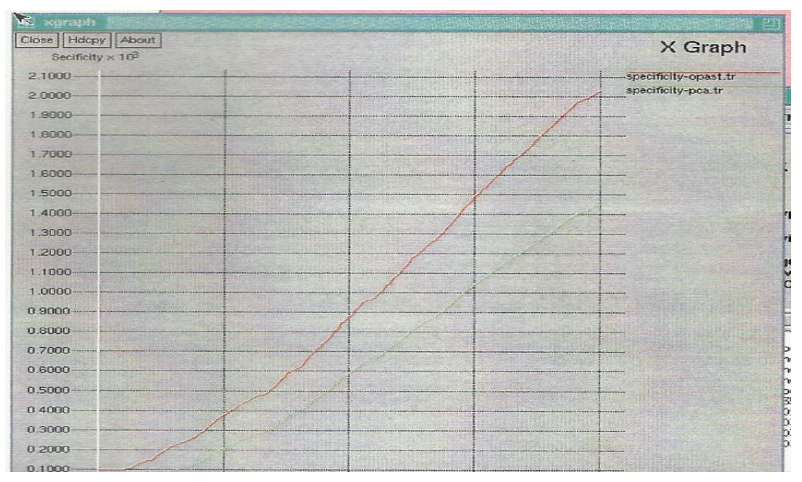

Figure 7. Throughput of the Network

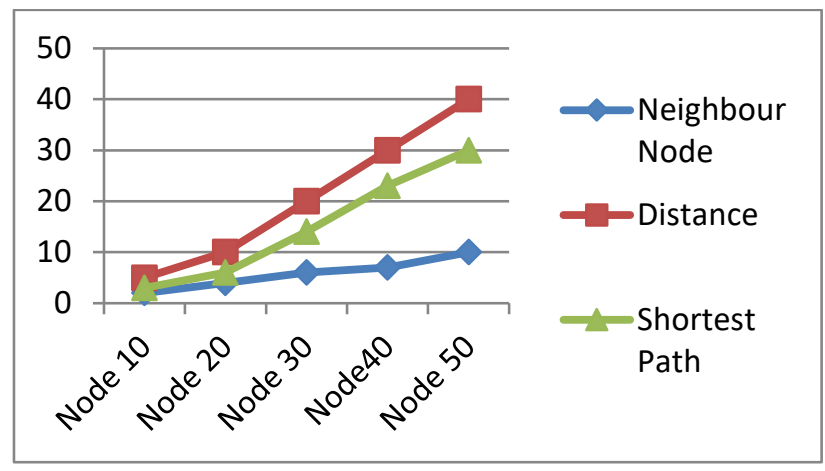

Figure 8. Comparison of Various Nodes

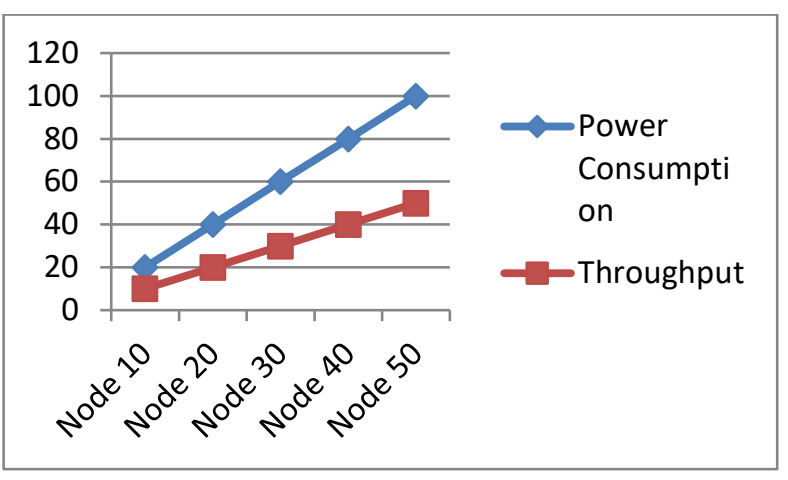

Figure 9. Power Consumption of various Nodes

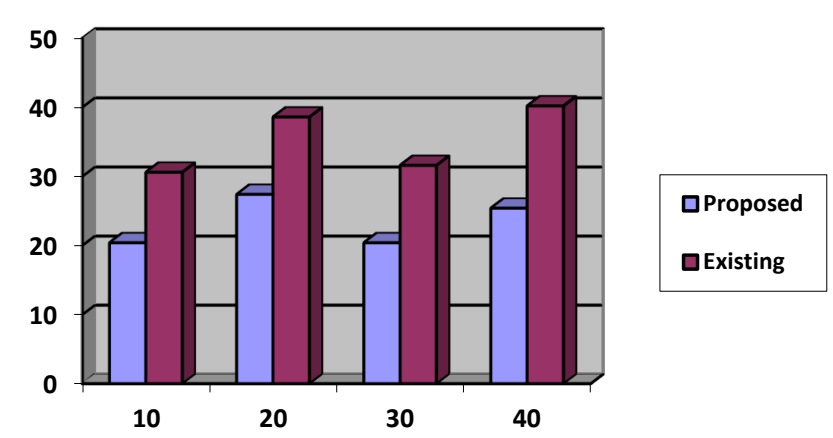

Figure 10. Power Consumption of the Proposed and Existing Network

\section{Conclusion and Future Scope}

PSO algorithm has been a popular technique is used to solve optimization problems in WSNs due to its easy, quality, fast 
coverage and insignificant computational burden. The simulation result shows that PSO produces good performance when compared to the other conventional algorithms. WSN network has challenge of reduced life time through network with the support like energy consumption of nodes is higher. The proposed method is used to analyse the various parameters like distance, power consumed and trust value by using PSO technique classifies the given sensor nodes into F and H Best Node. Future research on PSO method in WSN applications like: 1) Real world applications. 2) Development of PSO in hardware. 3) Development of parameters based on the network.

\section{References}

[1] Tian, D., Georganas, M.D.: " A coverage-preserving node scheduling scheme for large wireless sensor networks ". WSNA'02: Proc. First ACM Int. Workshop on Wireless Sensor Networks and Applications, 2002, pp. 32-41.

[2] Cardei, M., Thai, M.T., Yingshu, L., Weili, W.: “ Energyefficient target coverage in wireless sensor networks ". INFOCOM 2005: $24^{\text {th }}$ Annual Joint Conf. IEEE Computer and communications Societies, 2005, pp. 1976-1984.

[3] Huang, D.-F., Tseng, Y.-C.: " The coverage problem in a wireless sensor network “. WSNA'03: Proc. Second ACM Int. Conf. on Wireless Sensor Networks and Applications, 2003, pp. 115-121.

[4] Chih-fan, H., Mingyan, L.: " Network coverage using low dutycycled sensors: random \& coordinated sleep algorithms ". IPSN'04: Proc. Third Int. Symp. On Information Processing in Sensor Networks, 2004, pp. 433-442.

[5] Zhang, H., Wang, H., Feng, H.: " A distributed optimum algorithm for target coverage in wireless sensor networks". Asia-Pacific Conf. on Information Processing, 2009, pp. 144147.

[6] Fuad Bajaber, Irfan Awan.: Adaptive decentralized reclustering protocol for wireless sensor networks, Journal of computer and Systems sciences, doi:10.1016/j.jcss.2010.01.007.

[7] Yi-hua zhu, Wan-deng wu, Jian pan, Yi-ping tang.: An energy efficient data gathering algorithm to prolong lifetime of wireless sensor networks, Computer Communications 33, pp. 639-647 (2010).

[8] Fuad Bajaber, Irfan Awan.: Energy efficient clustering protocol to enhance lifetime of wireless sensor network, Journal of Ambient Intelligence and Human Computing 1, pp. 239-248 (2010).

[9] N.Dimokas, D.Katsaros,Y.Manolopoulos.: Energy-efficient distributed clustering in wireless sensor networks, Journal of Parallel and Distributed Computing 70, pp. 371-383 (2010).

[10] Jamal N. Al-Karaki, Raza Ul-Mustafa, Ahmed E. Kamal, "Data Aggregation in Wireless Sensor Networks - Exact and Approximate Algorithms"', Proceedings of IEEE Workshop on HighPerformance Switching and Routing (HPSR), USA.

[11] Suman Banerjee, Archan Misra, "Adapting Transmission Power for Optimal Energy Reliable Multi-hop Wireless Communication", Wireless Optimization Workshop (WiOpt'03), Sophia-Antipolis, France, March 2003.

[12] Niranjan Kumar Ray, Ashok Kumar Turuk, "Energy Efficient Techniques for Wireless Ad Hoc Network”,
[13] Shan Lin, Jingbin Zhang, Gang Zhou, Lin Gu, Tian He, and John A. Stankovic,"ATPC: Adaptive Transmission Power Control for Wireless Sensor Networks", SenSys '06 Proceedings of the 4th international conference on Embedded networked sensor systems, Pages 223-236

[14] S.G.Shirinivas, S.Vetrivel, N.M.Elango "Applications of Graph Theory in Computer Science - An Overview", International Journal of Engineering Science and Technology, Vol. 2(9), 2010, 4610-4621

[15] Bhupendra Gupta , Srikanth K Iyer , D Manjunath , "Topological Properties Of The One Dimensional Exponential Random Geometric Graph”, Random Structures \& Algorithms, Volume 32 , Issue 2 , 2008, pp: 181-204 [18] Chen Avin, "Random Geometric Graphs: An Algorithmic Perspective", Ph,D dissertation, University of California , Los Angeles , 2006 